\title{
Radiofrequency Ablation (Rafaelo Procedure) for the Treatment of Hemorrhoids: A Case Series in the United Kingdom
}

\author{
Sarah Hassan ${ }^{1}$, Daniel McGrath ${ }^{1}$, Richard Barnes ${ }^{2}$, Simon Middleton ${ }^{1}$ \\ ${ }^{1}$ Department of General Surgery, Royal Berkshire Hospital, Reading; ${ }^{2}$ Department of Anaesthetics, Royal Berkshire Hospital, Reading, United \\ Kingdom
}

Purpose: Hemorrhoidal disease remains a common condition that can have a significant effect on a patient's quality of life. Various methods have been introduced over the years; however, their overall success rates remain low. Although the traditional Milligan Morgan technique is effective, the associated pain level prevents it from being an attractive form of treatment. This study was devised to assess the safety and efficacy associated with a novel minimally invasive approach, radiofrequency ablation (RFA).

Methods: Forty-two patients underwent RFA at a single center, by 1 of 2 surgeons. This was performed under local anesthetic and sedation. Outcomes including postoperative pain levels, recurrence rates, and patient satisfaction scores were recorded and analyzed using medians and interquartile ranges

Results: The median postoperative pain score was 2.5/10 (interquartile range [IQR], $0-4.5$ ) and the overall patient satisfaction score was $9 / 10$ (IQR, 6.5-10). Recurrence rates (6-12 months following the procedure) were low at $12 \%$ and all patients reported milder symptoms at recurrence. There were no serious adverse complications

Conclusion: The results from this case series supports other limited data in concluding that RFA is a safe and effective method in the treatment of hemorrhoids and patients report a high level of satisfaction following

Keywords: Hemorrhoids; Radiofrequency ablation; Case series; Novel

\section{INTRODUCTION}

Hemorrhoids have a high prevalence among the international population, resulting in symptoms such as bleeding, itching, pain, and prolapse. It is estimated that $50 \%$ of the population has hemorrhoids by the age of 50 years [1]. Although prevention remains better than cure, once established, the condition can be difficult to treat effectively and definitively. Optimal treatment remains under debate and new techniques and instrumental tools continue

Received: Apr 19, 2021 - Revised: Jun 16, 2021 - Accepted: Jun 19, 2021 Correspondence to: Sarah Hassan, MBBS, FRCS

Department of General Surgery, Royal Berkshire Hospital, London Rd, Reading RG1 5AN, United Kingdom

Tel: +44-7809702132

E-mail:sah87@doctors.net.uk

ORCID: https://orcid.org/0000-0001-9958-2239

(C) 2021 The Korean Society of Coloproctology

This is an open-access article distributed under the terms of the Creative Commons Attribution NonCommercial License (https://creativecommons.org/licenses/by-nc/4.0) which permits unrestricted noncommercial use, distribution, and reproduction in any medium, provided the original work is properly cited. to be introduced to reduce postoperative pain and complications, including longer-term recurrences.

For grade I to II hemorrhoids, rubber band ligation (RBL) has been the initial mode of therapy. The results are superior to those of injection sclerotherapy [2] and the procedure can be carried out in the outpatient setting with no anesthetic. Single versus multiple RBL was investigated in 2 randomized studies and triple $\mathrm{RBL}$ was found to be more cost-effective with no increase in complication rate [2]. More recently, hemorrhoidal artery ligation (HAL) was introduced and the HubBLe Trial [3] demonstrated that there were fewer recurrences at 1 year with the HAL compared to RBL; however, the results were similar when compared with repeat RBL. There are not yet any studies that demonstrate long-term outcomes ( $>3$ years) with the HAL procedure.

In prolapsing grade III-IV hemorrhoids, the traditional Milligan Morgan hemorrhoidectomy (derived in 1937) has high success rates in minimizing recurrence; however, prolonged postoperative pain and recovery time are common complications [4]. This reduces overall patient satisfaction scores. Most patients 
would prefer a less invasive modality of treatment if given the option.

Radiofrequency ablation (RFA) is slowly being introduced as a potentially faster and less painful treatment than other methods. Radiofrequency is a type of electrical energy. RFA uses heat made by radio waves to thermally destroy tissue cells. The alternating current generates changes in the direction of ions within the tissue fluid. This creates ionic agitation and frictional heating, leading to coagulative necrosis of tissue. Long-term, in hemorrhoidal disease, this achieves plication of anorectal mucosa and improved symptoms. Although RFA is a novel approach for hemorrhoidal disease, it has been successfully used to treat other conditions such as varicose veins and Barrett's esophagus/dysplasia. Studies looking at outcomes of RFA in hemorrhoidal disease are limited. This is the largest case series reported in the United Kingdom, with a total of 42 patients who were treated at a single site, by 1 of 2 consultant colorectal surgeons.

\section{METHODS}

This study included 42 patients who underwent the Rafaelo Procedure (Modern Aesthetics Solutions Ltd., Elstree, UK) which uses the established technology of RFA for grade I to III hemorrhoids in a hospital by 1 of 2 colorectal consultants. The surgical method was standardized between both consultants performing the procedure and is described below. The treatment was performed under local anesthetic with minimal propofol sedation in the left lateral position. All procedures were carried out as day cases and patients were sent home with simple analgesia including 7 days of paracetamol + /- ibuprofen.

Prior to the procedure, the standard patient data was gathered including age, sex, comorbidities, symptoms, and their impact on

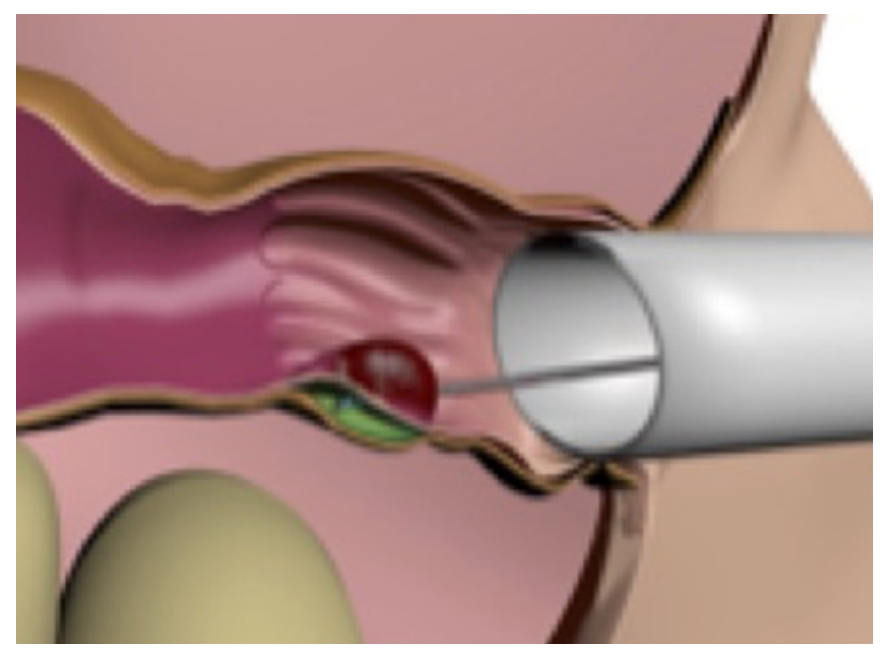

Fig. 1. Injection of local anesthetic into the submucosal plane of the hemorrhoid. Permission for image taken from Mark Plass at Modern Aesthetics Solution Ltd. (Elstree, UK). patient quality of life (QoL). Intraoperative data including the number of hemorrhoids treated, hemorrhoidal positions, energy levels used, and complications were all recorded for each patient. The dataset was statistically analyzed using medians and interquartile ranges. All the patients consented for inclusion into the study.

\section{Rafaelo Procedure}

A lubricated proctoscope is inserted into the anal canal, allowing good visualization of the hemorrhoids. Local anesthetic is inserted into the submucosal plane of each hemorrhoidal tissue (Fig. 1). This provides a fluid barrier to protect the internal anal sphincters against the transmission of heat, as well as providing a local anesthetic effect. A specially designed probe (Rafaelo HPR45i) connected to a radiofrequency generator is then inserted into hemorrhoid causing the tissue to heat up, and hemorrhoid to become necrotic and shrink (Fig. 2). The HPR45i probe allows good visualization of the tissues and the radiofrequency signal to be applied onto the tip of the probe when the generator is activated. The length of the probe measures $210 \mathrm{~mm}$ and the tip measures $10 \times 2 \mathrm{~mm}$.

\section{Follow-up}

All patients were followed up 6 to 8 weeks postoperatively in the outpatient clinic, and 6 to 12 months later by telephone consultation. At the latter stage, a patient questionnaire regarding postoperative pain level and procedure satisfaction score were recorded using the Likert scale.

\section{RESULTS}

Forty-two patients were treated with RFA between March 2017

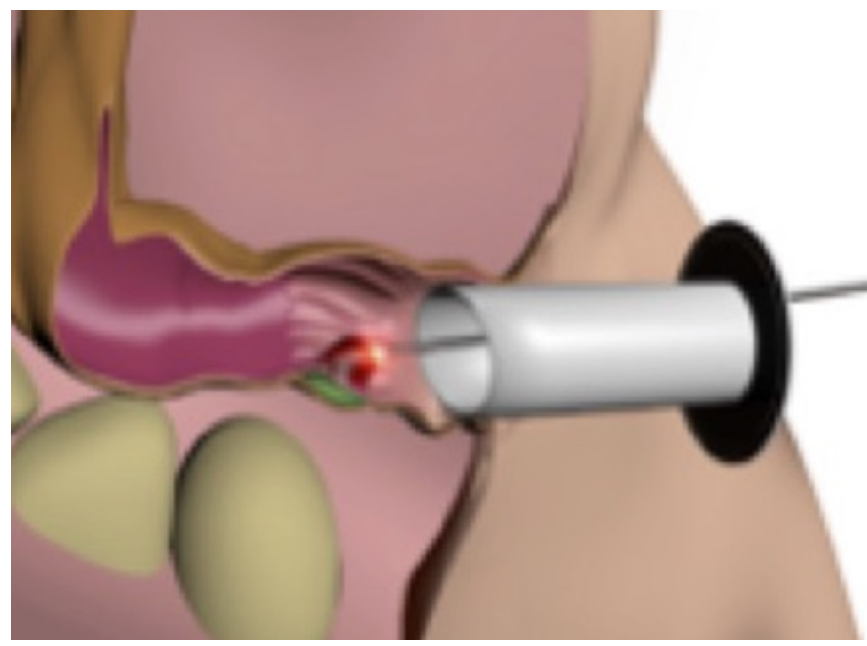

Fig. 2. Application of radiofrequency ablation into the hemorrhoid. Permission for image taken from Mark Plass at Modern Aesthetics Solution Ltd. (Elstree, UK). 
Table 1. Patient demographics

\begin{tabular}{lc}
\hline Demographic & Data \\
\hline No. of patients & 42 \\
Sex & \\
$\quad$ Male & $27(64.3)$ \\
Female & $15(35.7)$ \\
Age (yr) & $43(24-75)$ \\
Hemorrhoid grade & \\
I & $5(11.9)$ \\
॥ & $22(52.4)$ \\
III & $15(35.7)$ \\
Previous treatment & \\
Phenol injection & $5(11.9)$ \\
Rubber band ligation & $3(7.1)$ \\
Hemorrhoid artery ligation & $9(21.4)$ \\
\hline
\end{tabular}

Values are presented as number only, number (\%), or mean (range).

Table 2. Treatment details

\begin{tabular}{lc}
\hline Variable & Data \\
\hline No. of hemorrhoids treated & \\
1 & $2(4.8)$ \\
2 & $15(35.7)$ \\
3 & $25(59.5)$ \\
Energy applied (KJ) & 1,640 \\
Time of energy application (sec) & 70 \\
\hline
\end{tabular}

Values are presented as number of patients (percent) or mean value.

and December 2018 at Dunedin Hospital in Reading, United Kingdom. Patient demographics are listed in Table 1. Number and grade of hemorrhoids treated, mean energy levels, and time of energy application are shown in Table 2. The 2 main symptoms that patients complained of were bleeding $(28,66.7 \%)$ and pain/ discomfort $(29,69.0 \%)$. Other symptoms included mucus discharge $(1,2.4 \%)$, itching $(10,23.8 \%)$, prolapse $(4,9.5 \%)$, and urgency/incontinence of feces $(2,4.8 \%)$. When asked to quantify the effect of these symptoms on QoL using the Likert scale $(0-10,10$ being maximum impact on QoL), the median score was 7 (IQR, $6-8.25)$.

Following the procedure, the median pain score $(0-10,10$ being maximum pain) was 2.5 (IQR, 0-4.5) and this lasted 5 days on average. The patients of $33 \%$ required simple analgesia postoperatively. The patients of $38 \%$ reported no pain in both the immediate and initial postoperative weeks following surgery. Nine patients had already undergone previous HAL and comparatively, the median associated pain score was reported as $8 / 10$. Overall, the median satisfaction score with RFA was 8.4/10 (IQR, 6.5-10) (Table 3).
Table 3. The outcomes of the patients' symptoms and quality of life (QoL)

\begin{tabular}{lc}
\hline Variable & Outcome \\
\hline Symptom & 28 \\
Bleeding & 29 \\
Pain/discomfort & 1 \\
Discharge & 4 \\
Prolapse & 2 \\
Incontinence & 10 \\
Itching & \\
QoL score ${ }^{\text {a }}$ & $2 / 25^{\mathrm{b}}$ \\
$0-3$ & $16 / 13^{\mathrm{b}}$ \\
$4-7$ & $24 / 4^{\mathrm{b}}$ \\
$8-10$ & \\
Patient satisfaction score & \\
$0-2$ & 3 \\
3,4 & 0 \\
5,6 & 5 \\
7,8 & 8 \\
9,10 & 26 \\
\hline
\end{tabular}

${ }^{\mathrm{a}} 0=$ Not affected by symptoms, $10=$ affected daily by symptoms. ${ }^{\text {bPretreatment } /}$ posttreatment. ${ }^{\circ} 0=$ very unsatisfied, $10=$ very satisfied.

There were no significant postoperative complications. The readmission rate was $4.8 \%$. One patient was admitted for postoperative bleeding that settled without intervention, and 1 patient was admitted for pain management. Neither required an overnight hospital stay.

\section{DISCUSSION}

The purpose of this study was to test the safety and efficacy of the Rafaelo Procedure in a single cohort of patients with grade I to III hemorrhoids. This is the largest case series reported in the United Kingdom. The results illustrate a median pain score of $2.5 / 10$ with only $1 / 3$ patients requiring postoperative analgesia. Only 1 patient returned to the hospital with pain that was not controlled with conventional analgesia. Patients were followed up at 6 to 12 months to allow a reasonable period of time for symptom recurrence. Five patients (11.9\%) required further operative management within this period; however, all but 1 of these patients described milder symptoms at recurrence. All patients who required further treatment had grade III hemorrhoidal disease. The reasons for recurrence in these patients were felt to be multifactorial including high-grade disease, and lifestyle factors, such as dietary intake and infrequent bowel motions. The Rafaelo Procedure can be performed in stages for larger hemorrhoids. In conclusion, within this study group, the Rafaelo Procedure has been used 
safely in both primary and recurrent hemorrhoidal disease with no serious complications.

The literature was reviewed and compared to the results of this study. A recent case series in Cologne looked at 102 patients with grade III hemorrhoids undergoing the Rafaelo Procedure [5]. Their results also support the procedure being safe with no undesired effects. Their patient symptom score dropped from 9.9 to 1.1 after 3 months and pain levels were similar to our reported patient cohort. A polish center has also used the Rafaelo probe in a total of 4,000 interventions with no reported adverse complications (not yet published) [5]. The long-term results of both studies are not yet known. A more recent publication of results in the United Kingdom (Eddama et al. [6]) reports results in 27 patients and has shown a significant reduction in symptoms postprocedure. Their satisfaction scores match those found in our patient cohort.

Much of the other data regarding RFA in hemorrhoidal disease has been published in India. Two randomized trials were conducted; one comparing RFA with band ligation [7] and the other comparing RFA with the Milligan Morgan technique [8]. Both studies were small, with patient numbers under 60 . However, they both concluded that RFA is a safe method of treatment for patients with grade II to III hemorrhoids. They also reported lower postoperative pain scores and no serious adverse complications of RFA were documented, such as anal stenosis, incontinence, or wound sepsis.

The strengths of this study include a reasonable-sized case series, with a standardized operative method carried out by a limited number of surgeons. This reduces variability in technique. Clinic follow-up was also standardized, and individual telephone consultations were made to avoid losing patient to follow up, which enables a complete dataset for analysis. Limitations include possible recall bias, the fact this is a single centered study, and that there is no comparable treatment group.

This study supports other limited data in assurance that RFA is a safe and acceptable method of treatment for hemorrhoidal disease. Although the satisfaction scores are promising, further randomized controlled trials are needed to confirm the true efficacy of this method. A further trial is currently being conducted within this same center to assess pain levels and satisfaction scores with RFA treatment under local anesthetic without sedation in the outpatient setting.

\section{CONFLICT OF INTEREST}

No potential conflict of interest relevant to this article was reported.

\section{REFERENCES}

1. Ray-Offor E, Amadi S. Hemorrhoidal disease: predilection sites, pattern of presentation, and treatment. Ann Afr Med 2019;18:126.

2. Brown SR. Haemorrhoids: an update on management. Ther Adv Chronic Dis 2017;8:141-7.

3. Brown SR, Tiernan JP, Watson AJ, Biggs K, Shephard N, Wailoo AJ, et al. Haemorrhoidal artery ligation versus rubber band ligation for the management of symptomatic second-degree and third-degree haemorrhoids (HubBLe): a multicentre, open-label, randomised controlled trial. Lancet 2016;388:356-64.

4. Lohsiriwat V. Treatment of hemorrhoids: a coloproctologist's view. World J Gastroenterol 2015;21:9245-52.

5. Schäfer H, Tolksdorf S, Vivaldi C. Radiofrequency ablation (Rafa$\mathrm{elo}^{\circledR}$ procedure) for the treatment of prolapsing stage III haemorrhoids-technique and initial results. Coloproctology 2018;40:20410 .

6. Eddama MM, Everson M, Renshaw S, Taj T, Boulton R, Crosbie J. Radiofrequency ablation for the treatment of haemorrhoidal disease: a minimally invasive and effective treatment modality. Tech Coloproctol 2019;23:769-74.

7. Gupta PJ. Radiofrequency coagulation versus rubber band ligation in early hemorrhoids: pain versus gain. Medicina (Kaunas) 2004;40:232-7.

8. Gupta PJ. Randomized trial comparing in-situ radiofrequency ablation and Milligan-Morgan hemorrhoidectomy in prolapsing hemorrhoids. J Nippon Med Sch 2003;70:393-400. 